\title{
DYNAMO REGIMES WITH A NONHELICAL FORCING
}

\author{
Pablo D. Mininni, ${ }^{1}$ Yannick Ponty, ${ }^{2}$ David C. Montgomery ${ }^{3}$ Jean-Francois Pinton, $^{4}$ \\ Helene Politano, ${ }^{2}$ and Annick Pouquet ${ }^{1}$ \\ Received 2004 December 2; accepted 2005 February 24
}

\begin{abstract}
A three-dimensional numerical computation of magnetohydrodynamic dynamo behavior is described. The dynamo is mechanically forced with a driving term of the Taylor-Green type. The magnetic field development is followed from negligibly small levels to saturated values that occur at magnetic energies comparable to the kinetic energies. Although there is locally a nonzero helicity density, there is no overall integrated helicity in the system. Persistent oscillations are observed in the saturated state for not-too-large mechanical Reynolds numbers, oscillations in which the kinetic and magnetic energies vary out of phase but with no reversal of the magnetic field. The flow pattern exhibits considerable geometrical structure in this regime. As the Reynolds number is increased, the oscillations disappear and the energies become more nearly stationary, but retain some unsystematically fluctuating turbulent time dependence. The regular geometrical structure of the fields gives way to a more spatially disordered distribution. The injection and dissipation scales are identified, and the different components of energy transfer in Fourier space are analyzed, particularly in the context of clarifying the role played by different flow scales in the amplification of the magnetic field. We observe that small and large scales interact and contribute to the dynamo process.
\end{abstract}

Subject headings: magnetic fields - MHD

Online material: color figures

\section{INTRODUCTION}

Evidence of the existence of magnetic fields is known in many astronomical objects. These fields are believed to be generated and sustained by a dynamo process (e.g., Moffatt 1978), and often these objects are characterized by the presence of largescale flows (such as rotation) and turbulent fluctuations. These two ingredients are known to be often associated with magnetohydrodynamic dynamos. In recent years, significant advances have been made either studying large-scale flow dynamos in the kinematic approximation or using direct numerical simulations to study turbulent amplification of magnetic fields in simplified geometries.

In a previous paper (Ponty et al. 2005), a study of the selfgeneration of magnetic fields in a turbulent conducting fluid was reported. The study was computational and dealt mainly with the effects of lowering the magnetic Prandtl number $P_{M}$ of the fluid (ratio of kinematic viscosity to magnetic diffusivity). The velocity field was externally excited by a forcing term on the right-hand side of the equation of motion whose geometry was that of what has come to be called the Taylor-Green vortex (Taylor \& Green 1937; Morf et al. 1980; Pelz et al. 1985; Nore et al. 1997; Marié et al. 2003; Bourgoin et al. 2004). The regime of operation was one of kinetic Reynolds number $\gg 1$ (so that the fluid motions were turbulent), and the emphasis was on how large the magnetic Reynolds numbers had to be for the infinitesimal magnetic fields to be amplified and grow to macroscopic values.

Here, we want to describe and stress another aspect of the Taylor-Green dynamo. In particular, we have found computation-

\footnotetext{
1 National Center for Atmospheric Research, P.O. Box 3000, Boulder, CO 80307-3000.

2 Laboratoire Cassiopée, CNRS UMR6203, Observatoire de la Côte d'Azur, BP 4229, F-06034 Nice Cedex 04, France.

3 Department of Physics and Astronomy, 6127 Wilder Laboratory, Dartmouth College, Hanover, NH 03755.

4 Laboratoire de Physique, CNRS UMR5672, École Normale Supérieure de Lyon, 46 Allée d'Italie, 69007 Lyon, France.
}

ally that it has an oscillatory regime, for not too large a Reynolds number, in which energy is passed back and forth regularly between the mechanical motions and the magnetic excitations in a way we believe to be new. Out of the velocity field emerges a geometrically regular, time-averaged pattern involving coherent magnetic and mechanical oscillations.

As the Reynolds number is increased, the resulting flow has a well-defined large-scale pattern and nonhelical turbulent fluctuations. In this case, the oscillations disappear, and the magnetic field grows at scales both larger and smaller than the integral scale of the flow. After the nonlinear saturation of the dynamo, velocity field fluctuations are partially suppressed, and a magnetic field with a spatial pattern reminiscent of the low Reynolds number case can be identified. This complex evolution of the magnetic field can be understood by studying the role played by the energy transfer in Fourier space.

In $\oint 2$, we describe the numerical experiments and outline a typical time history of the development of an oscillatory dynamo. We then go on to show how, by increasing the Reynolds number, the oscillatory behavior can be suppressed. In $\S 3$, we make use of color displays of the field quantities to demonstrate the cycle of the oscillation and to reveal the intriguing and complex varying three-dimensional pattern that characterizes it. The pattern, although regular, is difficult to see through completely in physical terms. Finally, $\S 4$ suggests some precedents, provides a partial explanation, and considers other similar situations in which such coherence may or may not be expected to emerge out of turbulent disorder.

\section{THE COMPUTATION}

The Taylor-Green vortex is a flow with an initial periodic velocity field

$$
\boldsymbol{v}_{\mathrm{TG}}\left(k_{0}\right)=\left[\begin{array}{c}
\sin \left(k_{0} x\right) \cos \left(k_{0} y\right) \cos \left(k_{0} z\right) \\
-\cos \left(k_{0} x\right) \sin \left(k_{0} y\right) \cos \left(k_{0} z\right) \\
0
\end{array}\right]
$$


and was originally introduced as an initial condition that, although highly symmetric, would lead to the rapid development of small spatial scales (Taylor \& Green 1937). We introduce it here on the right-hand side of the magnetohydrodynamic (MHD) equation on motion for the velocity field $\boldsymbol{v}$ :

$$
\frac{\partial \boldsymbol{v}}{\partial t}+\boldsymbol{v} \cdot \nabla \boldsymbol{v}=-\nabla \mathcal{P}+\boldsymbol{j} \times \boldsymbol{B}-\nu \nabla \times \boldsymbol{\omega}+F \boldsymbol{v}_{\mathrm{TG}},
$$

where $\boldsymbol{B}$ is the magnetic field, advanced by

$$
\frac{\partial \boldsymbol{B}}{\partial t}+\boldsymbol{v} \cdot \nabla \boldsymbol{B}=\boldsymbol{B} \cdot \nabla \boldsymbol{v}-\eta \nabla \times \boldsymbol{j} .
$$

Equations (2) and (3) are to be solved pseudospectrally. The current density is $\boldsymbol{j}=\nabla \times \boldsymbol{B}$ (we use the common dimensionless Alfvénic units), $F$ is a forcing amplitude, and $k_{0}=2(2 \pi / L)$, where $L=2 \pi$ is chosen as the basic periodicity length in all three directions. In the incompressible case, $\nabla \cdot \boldsymbol{v}=0$ and $\nabla \cdot \boldsymbol{B}=$ $0 ; \nu^{-1}$ and $\eta^{-1}$ are (dimensionless) mechanical and magnetic Reynolds numbers, since we take as characteristic velocity and length $U_{0}=1$ and $L_{0}=1$ leading to an eddy turnover time of order unity; and $\mathcal{P}$ is the dimensionless pressure, normalized by the (uniform) mass density.

The strategy is to turn on a nonzero force $F$ at $t=0$ and allow the code to run for a time as a purely Navier-Stokes code, with the $\boldsymbol{B}$ and $\boldsymbol{j}$ fields set at zero. The initial velocity field is given by

$$
\boldsymbol{v}_{0}=-\frac{F}{\nu} \nabla^{-2} \boldsymbol{v}_{\mathrm{TG}}
$$

and the amplitude of $F$ is set to obtain an initial unitary rms velocity. As the system evolves, more modes are excited and the dissipation increases. To maintain the kinetic energy at the same level, the amplitude of the force is controlled during the hydrodynamic simulation to compensate the dissipation. At each time $t$, the energy injection rate,

$$
\epsilon=F(t) \int \boldsymbol{v} \cdot \boldsymbol{v}_{\mathrm{TG}} d^{3} x,
$$

and the enstrophy,

$$
\Omega=\frac{1}{2} \int(\nabla \times v)^{2} d^{3} x,
$$

are computed, and the amplitude of the external force needed to overcome dissipation is computed as

$$
F^{*}=\frac{2 \nu \Omega F(t)}{\epsilon} .
$$

The response of the velocity field to the change in the external force has a certain delay, and to avoid spurious fluctuations the average value $\left\langle F^{*}\right\rangle$ of this quantity is computed for both the last nine time steps and the averaged error in the energy balance $\mathcal{E}=\langle 2 \nu \Omega-\epsilon\rangle$. Finally, the amplitude of the external force at time $t+\Delta t$ is updated as

$$
F(t+\Delta t)=0.9 F^{*}+\left(0.1\left\langle F^{*}\right\rangle+0.01 \mathcal{E}\right) / 9 .
$$

Once a stationary state is reached, the last computed amplitude of the force can be used to restart the simulation with constant force instead of constant energy. In this case, the energy fluctuates around its original value, and the rms velocity averaged in time is unity. This value of the rms velocity and the integral length scale $L_{\text {int }}$ of the resulting flow are used to defined the Reynolds numbers in the following sections. For a different scheme to compensate the dissipation, see e.g., Archontis et al. (2003).

Once the stationary kinetic state is reached, the magnetic field is seeded with randomly chosen Fourier coefficients and allowed to amplify. All the MHD simulations are done with constant force, and the amplitude $F$ is obtained as previously discussed. The initial magnetic field is nonhelical, with the magnetic energy smaller than the kinetic energy at all wavenumbers, and a spectrum satisfying a $k^{2}$ power law at large scales and an exponential decay at small scales. A previous paper has described the "kinematic dynamo" regime, in which the magnetic excitations, while growing, are too small to affect the velocity field yet (Ponty et al. 2005). In particular, a threshold curve for magnetic field amplification was constructed in the plane whose axes are a magnetic Prandtl number, $P_{M} \equiv \nu / \eta$, and a magnetic Reynolds number. As $P_{M}^{-1}$ increases, there is a sharp rise in the dynamo threshold, followed by a plateau. Here, the purpose is to follow the evolution of $\boldsymbol{B}$ out of the kinematic regime and observe whatever saturation mechanisms may set in.

\section{COMPUTATIONAL RESULTS}

Table 1 summarizes the parameters of the four runs we have carried out. Runs A and $\mathrm{A}^{\prime}$ have relatively low mechanical and magnetic Reynolds numbers ( $\sim 40$, based on the integral length scale and the rms velocity), while runs $\mathrm{B}$ and $\mathrm{B}^{\prime}$ have mechanical Reynolds numbers of $R_{V}=675$. The magnetic Reynolds numbers $R_{M}$ for runs $\mathrm{A}$ and $\mathrm{A}^{\prime}$ are 33.7 and 37.8, respectively, while those for B and $\mathrm{B}^{\prime}$ were 240.2 and 270 , respectively. These values of $R_{M}$ were in all cases above the previously determined thresholds (Ponty et al. 2005) for magnetic field growth (see Fig. 1). Note that $R_{M}$ for runs $\mathrm{A}$ and $\mathrm{B}$ is $6 \%$ above the threshold, while runs $\mathrm{A}^{\prime}$ and $\mathrm{B}^{\prime}$ are $20 \%$ above the threshold. We chose $k_{0}=2$ in all cases, so that the kinetic energy spectrum peaks at $k=k_{0} \sqrt{3} \approx 3$. As previously mentioned, the amplitude of the external force was constant during the MHD simulation, and given by $F=0.926$ in runs $\mathrm{A}$ and $\mathrm{A}^{\prime}$, and $F=0.37$ in runs $\mathrm{B}$ and $\mathrm{B}^{\prime}$.

The definitions of the Taylor microscale $\lambda$ and the integral scale $L_{\text {int }}$ in Table 1 are based on the kinetic energy spectrum $E_{V}(k)$,

$$
\begin{aligned}
L_{\mathrm{int}} & =2 \pi \int k^{-1} E_{V}(k) d k / \int E_{V}(k) d k, \\
\lambda & =2 \pi\left[\int E_{V}(k) d k / \int k^{2} E_{V}(k) d k\right]^{1 / 2} .
\end{aligned}
$$

Note that the usual turbulent relationships between these two quantities based on the mechanical Reynolds number do not hold, since the energy spectra of runs $A$ and $\mathrm{A}^{\prime}$ do not display an inertial range.

\subsection{Low Reynolds Numbers and Close to the Threshold}

The behavior at saturation is very different for the high and low Reynolds numbers. The histories of the energies for runs A and B (both 6\% above threshold) are displayed in Figure 2. The upper two curves are the kinetic energies of these runs, a solid line for run A and a dotted line for run B. The lower two curves are the magnetic energies, with the same conventions. The origin of time is chosen from the moment when the seed magnetic fields are introduced.

It is clear that saturation is achieved unsystematically for the high $R_{V}$ run $\mathrm{B}$, with the resulting magnetic energy being smaller 
TABLE 1

Simulations

\begin{tabular}{|c|c|c|c|c|c|c|c|c|c|c|}
\hline Run & $\nu$ & $\eta$ & $R_{V}$ & $R_{M}$ & $R_{M}^{c \text { a }}$ & $P_{M}^{\mathrm{b}}$ & $L_{\text {int }}$ & $\lambda^{\mathrm{c}}$ & $N^{\mathrm{d}}$ & $\Delta^{\mathrm{e}}$ \\
\hline А & $5 \times 10^{-2}$ & $6 \times 10^{-2}$ & 40.5 & 33.7 & 31.7 & 0.83 & 2.02 & 1.69 & $64^{3}$ & 6 \\
\hline В & $2 \times 10^{-3}$ & $5.62 \times 10^{-3}$ & 675 & 240.2 & 226.4 & 0.35 & 1.35 & 0.6 & $256^{3}$ & 6 \\
\hline $\mathrm{A}^{\prime}$ & $5 \times 10^{-2}$ & $5.35 \times 10^{-2}$ & 40.5 & 37.8 & 31.7 & 0.93 & 2.02 & 1.69 & $64^{3}$ & 20 \\
\hline $\mathrm{B}^{\prime} \ldots \ldots \ldots$ & $2 \times 10^{-3}$ & $5 \times 10^{-3}$ & 675 & 270 & 226.4 & 0.4 & 1.35 & 0.6 & $256^{3}$ & 20 \\
\hline
\end{tabular}

Note.-The Reynolds numbers $R_{V}$ and $R_{M}$ are based on the integral length scale $L_{\text {int }}$.

${ }^{\text {a }}$ Critical Reynolds number obtained for the given $R_{V}$.

b The magnetic Prandtl number is $P_{M}=\nu / \eta$.

c Taylor scale.

${ }^{\mathrm{d}}$ Spatial grid resolution used in the simulation.

e Gives the percentage above threshold in $R_{M}$ for each simulation.

than the kinetic energy and both being in a statistically steady state. The solid lines associated with the lower Reynolds number run A, however, show a systematic, sharp oscillation in both energies, with the maxima of one almost coinciding with the minima of the other. This is clearly a significantly different behavior from the high $R_{V}$ case, and is only partially understood. Such out-of-phase oscillations have already been observed in the nonlinear regime in constrained geometries, for example, by using a quasi-geostrophic model for strongly rotating flows (Schaeffer 2004) or a 2.5-dimensional formulation for the Ekman layer instability (Ponty et al. 2001).

In the two simulations presented in Figure 2, $R_{M}$ is $6 \%$ larger than $R_{M}^{c}$, and the growth rates during the kinematic regime are similar. While $\eta$ is 1 order of magnitude smaller in run B than in run $\mathrm{A}$, the nonlinear saturation in both runs takes place at approximately the same time. In both runs the integral eddy turnover time is approximately the same. This contrasts with dynamos in flows with net helicity, in which the nonlinear saturation was shown to occur in a magnetic diffusion time (Brandenburg 2001) (the diffusion time based on the integral lengthscale of the flow is of the order of 60 for run A and 360 for run B). Note that although the flow generated by the Taylor-Green force is locally helical, the net helicity of the flow in the entire domain is zero.

The forcing term generating the flow from equations (2) and (3) is initially entirely in the horizontal $(x, y)$ directions. It is essentially a vortical flow whose phase oscillates with increasing $z$. The velocity field in equation (2) is not, however, a steady state, and vertical (z) components develop quickly,

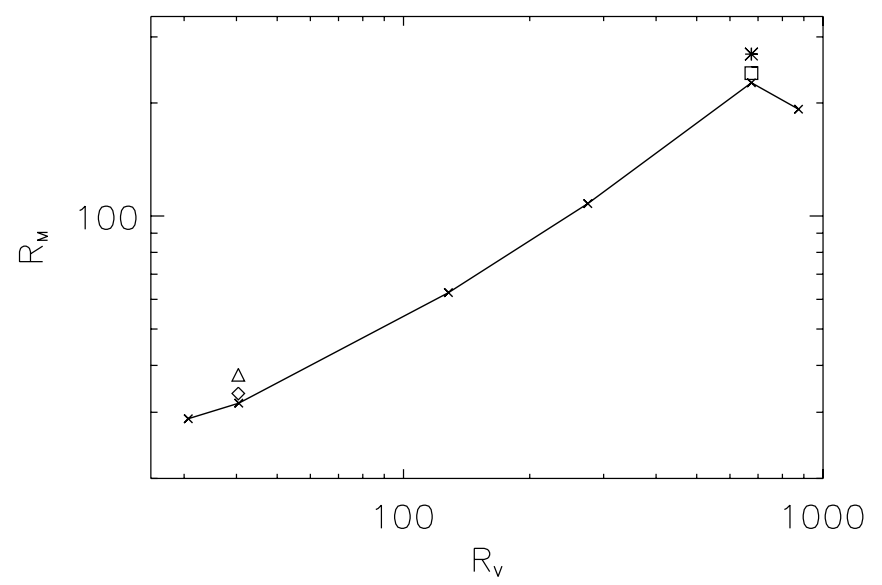

FIG. 1.-Critical magnetic Reynolds $R_{M}^{c}$ for dynamo action (solid line) inferred from direct numerical simulations (crosses), as a function of $R_{V}$ (Ponty et al. 2005). The position of the runs discussed in Table 1 are indicated by symbols: run A (diamond), $\mathrm{A}^{\prime}$ (triangle), B (square), and B' (asterisk). leading to an approximately meridional flow to be added to the toroidal one in each cell. A total streamline will resemble the shape of a wire wrapped around the outside of a doughnut, diagonally, which enters the hole of the doughnut at the bottom and emerges at the top. This flow shares similarities with the Cadarache and Wisconsin sodium experiments, and it has motivated several numerical studies of dynamo action at $P_{M}=1$. For a detailed description of the flow, we refer the reader to Nore et al. (1997) and Marié et al. (2003).

The amplification process for the magnetic field is difficult to visualize in this geometry. Field lines seem to be sucked into the hole of the doughnut and stretched and twisted in the process. The resulting amplified magnetic flux is then deposited and piled up in the horizontal planes between the cells. This flux, in turn, is the source of the field lines, which are further sucked into the holes in the doughnut and amplified. In the kinematic regime, but in a different geometry (including boundary conditions), the amplification of a magnetic field by a similar flow was also discussed in Marié et al. (2003) and Bourgoin et al. (2004).

Throughout the process, the rate of doing work by the magnetic field on the velocity field originates in the Lorentz force contribution, $-(\boldsymbol{j} \times \boldsymbol{B}) \cdot \boldsymbol{v}$. This energy input into the magnetic field is ohmically dissipated by the $\eta j^{2}$ integral. As the magnetic field grows, the fluid must work harder mechanically, because $\boldsymbol{j}$ and $\boldsymbol{B}$ are increasing. Since $F$ is constant, eventually a limit is

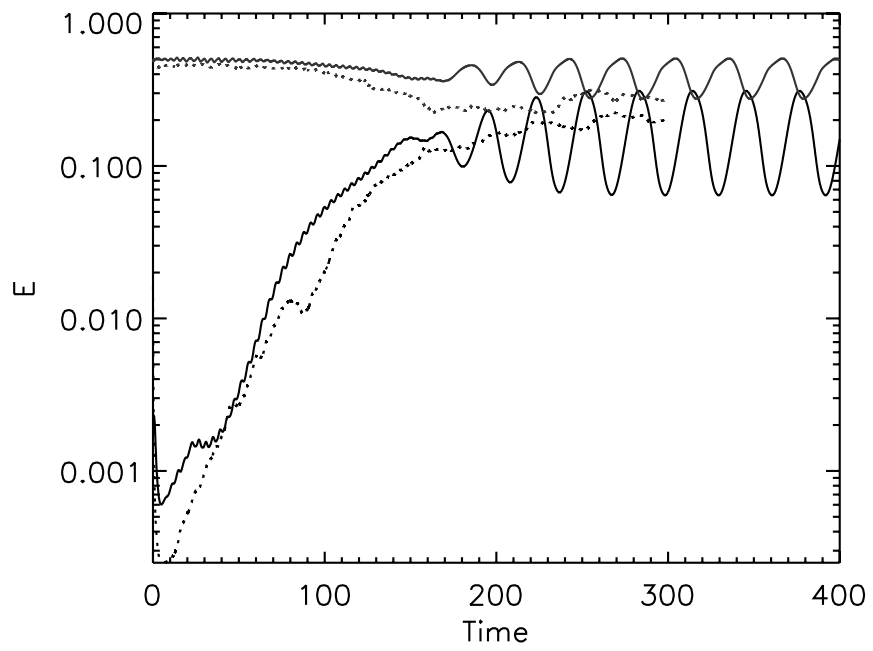

FIG. 2.-Time history of kinetic (upper curves) and magnetic energy (lower curves) for run A at low Reynolds (solid lines) and B at high Reynolds (dotted lines), both $6 \%$ above threshold. [See the electronic edition of the Journal for a color version of this figure.] 


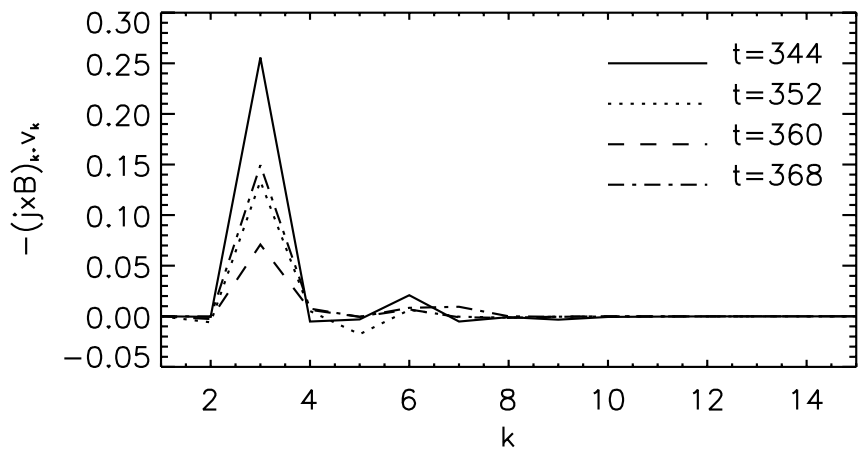

FIG. 3.-Fourier spectrum of $-(\widehat{\boldsymbol{j} \times \boldsymbol{B}})_{k} \cdot \hat{\boldsymbol{v}}_{k}^{*}$ for different times, for run $\mathrm{A}$, for one oscillation.

reached at which $\boldsymbol{v}$ can no longer transfer energy to $\boldsymbol{B}$ at its previous rate and slows down. At that point, the magnetic energy begins to be transferred in the reverse sense, so that $v$ grows again as $\boldsymbol{j}$ and $\boldsymbol{B}$ become weaker. The cyclic nature of the process ensues.

It is revealing to decompose $(\boldsymbol{j} \times \boldsymbol{B}) \cdot \boldsymbol{v}$ spectrally and plot the Fourier spectrum of $-(\boldsymbol{j} \times \boldsymbol{B})_{k} \cdot \hat{\boldsymbol{v}}_{k}^{*}$ as a function of $k$, where the asterisk denotes a complex conjugate and the real part is to be assumed, as shown in Figure 3 for run A. The peak near $k=$ 3 shows that this is the region where the mechanical work is being done to create the magnetic energy. The curve is plotted at four times during a complete oscillation, including $t=344$, when the magnetic energy is at its maximum during the cycle, and $t=360$, when the magnetic energy is at its minimum.

There is considerable structure to the flow for these low Reynolds number cases, anchored by the driving term in equation (2). Figure 4 shows instantaneous plots of the velocity field components along a vertical cut at $x=3 \pi / 8$ and $y=\pi / 4$, as functions of $z$ for run $\mathrm{A}$. This cut corresponds to a line in the $z$-direction displaced (in the $x-y$ plane) out of the center line of the vortices imposed by the external Taylor-Green force (corresponding to $x=y=\pi / 4)$. Plotted in Figure $4 a$ is $\left(v_{y}^{2}+v_{z}^{2}\right)^{1 / 2}$ versus $z$, and in Figure $4 b, v_{x}$ versus $z$. In both curves, four different times are shown. In this cut, $v_{x}$ corresponds to the amplitude of the toroidal flow associated with the vortices imposed by the forcing, while $\left(v_{y}^{2}+v_{z}^{2}\right)^{1 / 2}$ can be associated with the meridional flow previously defined. Note the mirror symmetries satisfied by the flow. As the oscillations evolve, not only is the amplitude of the flow changed, but the position of the maxima are also slightly displaced. The flow geometry is clarified in more detail in Figure 5.

Figures $5 a$ and $5 b$ exhibit cross-sectional plots of the velocity field in the plane $z=0$ at two different times for run A. The arrows show the directions of $v_{x}-v_{y}$, and the colors indicate the values of $v_{z}$, positive (light) or negative (dark), at the same locations. Note the 16 vortices imposed by the external TaylorGreen forcing with $k_{0}=2$. The amplitude of these vortices is modulated in $z$, and at $\pi / 2$ the same structure is obtained in the flow but with the vortices rotating in the opposite direction. Most of the stretching of the magnetic field takes place in these cells. Between these structures, at $z=\pi / 4$, stagnation points are present where the magnetic field piles up, as will be shown.

Figures $5 c$ and $5 d$ display similar plots at the plane $y=\pi / 4$, with $v_{x}, v_{z}$ indicated by the arrows, and $v_{y}$ by color. The regions of alternating color correspond to the cross section of the vortices imposed by the Taylor-Green forcing. The meridional flow can also be identified in these cross sections. However, note that this flow during the cycle is modified by the magnetic field in a
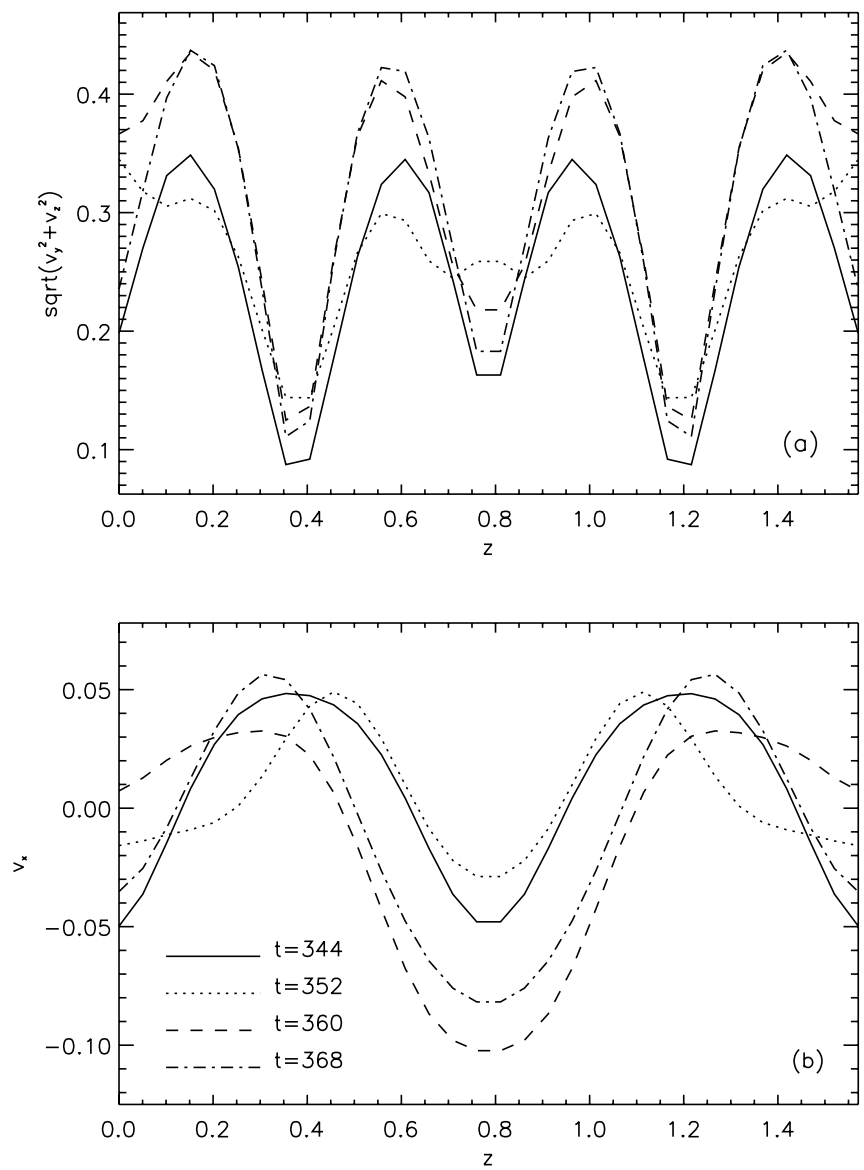

FIG. 4.-(a) $\left(v_{y}^{2}+v_{z}^{2}\right)^{1 / 2}$ and (b) $v_{x}$ as a function of $z$ at $x=3 \pi / 8$ and $y=\pi / 4$, and at different times for run A, for one oscillation.

more dramatic way than the toroidal flow. As shown in Figures 3 and 4, the Lorentz force mostly opposes the velocity field at large scales. The final effect of the magnetic field on the flow seems to be to suppress small-scale fluctuations, leaving a wellordered pattern. This effect is more dramatic at large $R_{V}$, as shown in $\S 3.2$.

Figures $6 a$ and $6 b$ show the magnetic field in the plane $z=0$ at the same times with the same conventions $\left(B_{x}, B_{y}\right.$ are arrows, $B_{z}$ is indicated by color), again for run A. The stretching of magnetic field lines by the toroidal flow can be observed in these sections. Figures $6 c$ and $6 d$ show the magnetic field in the plane $y=\pi / 4$ at the same times, and with the same plotting conventions. Note in dark and light colors the horizontal bars where most of the magnetic energy is concentrated. These regions correspond to stagnation planes of the external Taylor-Green forcing.

Finally, Figure 7 shows the magnetic field in the plane $z=$ $\pi / 4$ at different times for run A. This is a plane between rows of basic cells and is a candidate where the amplified flux "piles up" as previously indicated. It is apparent that the dynamical variation is much less in this plane during the cycle. Also in Figure 7 note the presence of locally "dipolar" structures (light and dark regions) centered in each of the Taylor-Green cells. These structures correspond to the almost uniform (and mostly concentrated in the $x-y$ plane) magnetic field being sucked into the hole of the doughnut given by the Taylor-Green force.

A more detailed picture of the dynamics of the forced TaylorGreen dynamo at low Reynolds numbers has eluded us, but it is imaginable that in less complex flows a more comprehensive understanding of the low $R_{V}$ nonhelical dynamo may be possible. 

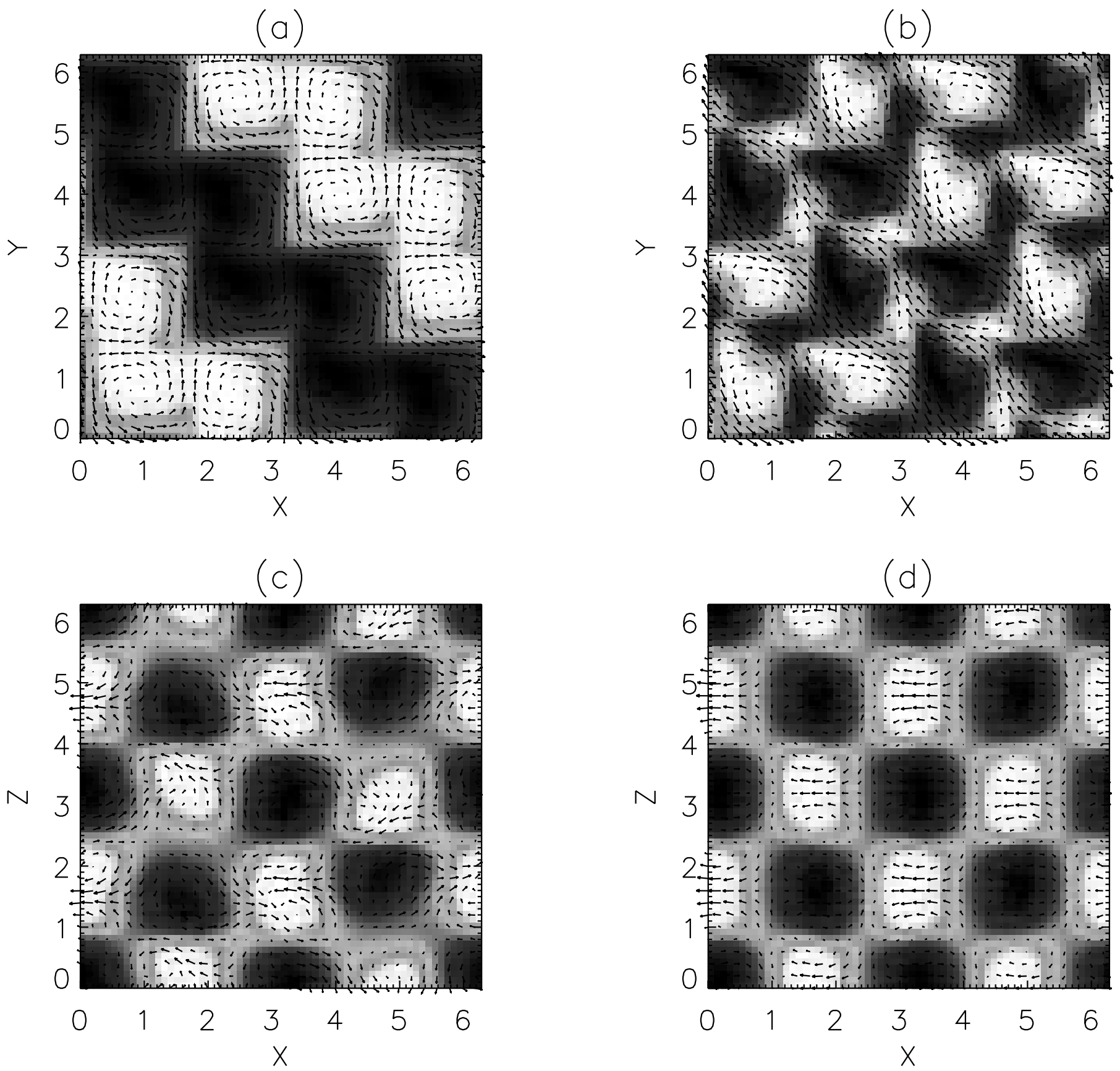

FIG. 5.-Upper panels: Cross-sectional plots of the velocity field in the plane $z=0$ at $(a) t=352$ and $(b) t=368$, for run A. The arrows show the directions of $v_{x}, v_{y}$, and the colors indicate the values of $v_{z}$, positive (light) or negative (dark). Lower panels: Cross-sectional plots of the velocity field in the plane $y=\pi / 4$ at (c) $t=352$ and $(d) t=368\left(v_{x}, v_{z}\right.$ is indicated by the arrows, and $v_{y}$ by the color). [See the electronic edition of the Journal for a color version of this figure.]

\subsection{High Reynolds Numbers and Farther from the Threshold}

Runs B and $\mathrm{B}^{\prime}$ involve higher Reynolds numbers and behave rather differently from runs $\mathrm{A}$ and $\mathrm{A}^{\prime}$. Figure 8 contrasts the time histories of the kinetic energies and magnetic energies for run $\mathrm{A}^{\prime}$ (solid lines) and run $\mathrm{B}^{\prime}$ (dashed lines), both $20 \%$ above threshold. The upper curves are kinetic energies and the lower curves are magnetic energies. It is clear that runs B and $\mathrm{B}^{\prime}$ saturate at a level of near equipartition and do not exhibit the oscillatory behavior seen in the lower Reynolds number runs. Run $\mathrm{A}^{\prime}$ retains a vestige of the periodic behavior, seen most clearly in the magnetic energy curve, which is quasi periodic or close to "chaotic." Note the overshooting of the magnetic energy for run $\mathrm{B}^{\prime}$ near $t \sim 150$, linked to the large drop in kinetic energy. Note also the similar growth rates (as for runs A and B), although the magnetic diffusivities differ again by almost an order of magnitude.

During the exponential period of the magnetic energy growth, it is of interest to note that the various Fourier modes all appear to be growing at the same rate in run $\mathrm{B}^{\prime}$ (the same effect is observed in run B). This can be seen by separating the Fourier space into "shells" of modes of the same width $\Delta k$. The time histories of these shells are plotted in Figure 9. In the inset, all the shells have been normalized to have the same amplitudes per $k$-mode at $t=4$, to show that the exponentiation rates up to about $t=30$ are the same or nearly so. This behavior is characteristic of small-scale dynamos (Kazantsev 1967; Brandenburg 2001). Note that after $t=30$ the shell with $k=2$ 

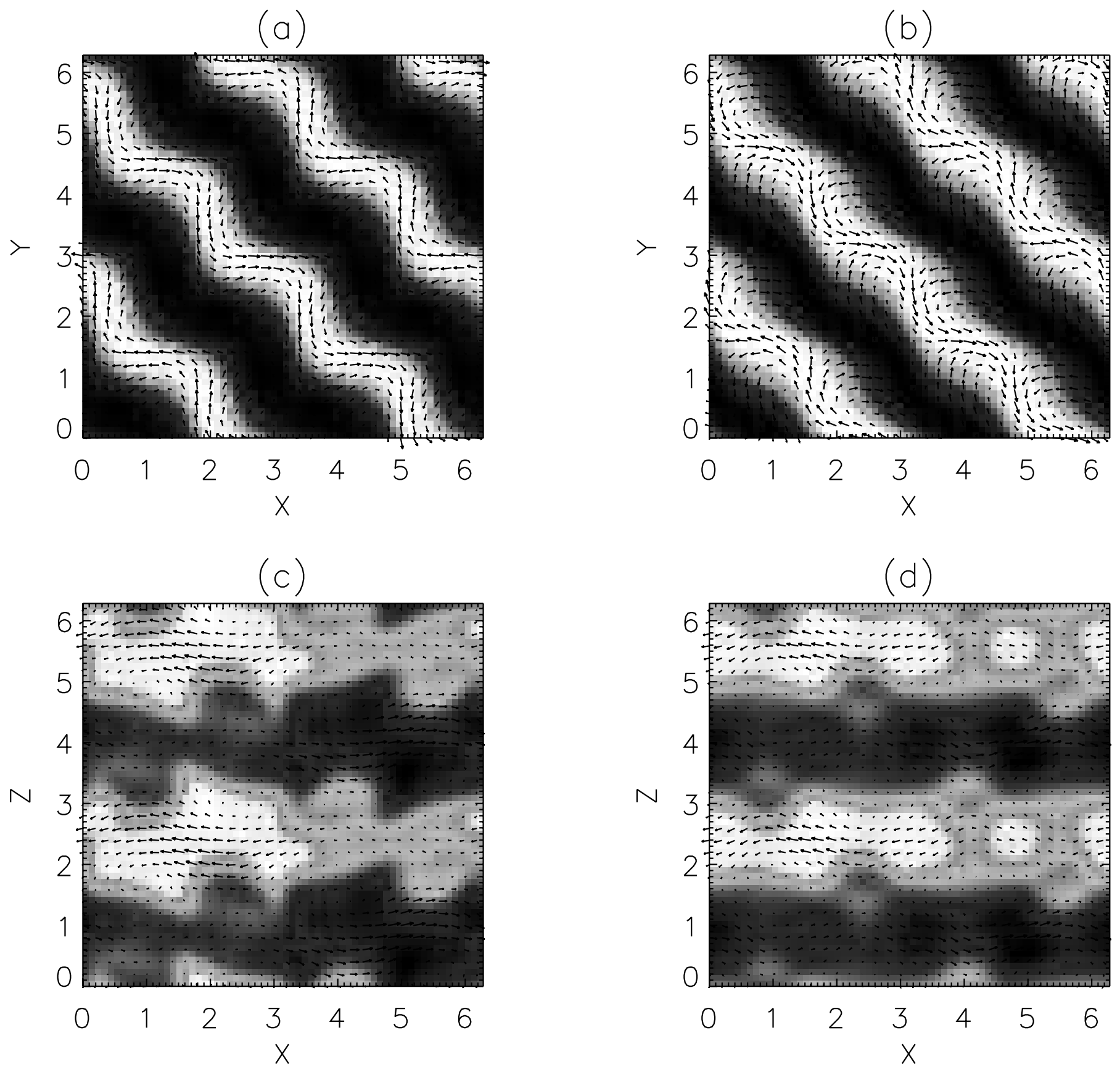

Fig. 6.-Upper panels: Cross-sectional plots of the magnetic field in the plane $z=0$ at $(a) t=352$ and $(b) t=368$, for run A. Lower panels: Cross-sectional plots of the magnetic field in the plane $y=\pi / 4$ at $(c) t=352$ and $(d) t=368$. Same conventions as in Fig. 5. [See the electronic edition of the Journal for a color version of this figure.]

seems to start growing faster than the small-scale modes. Shortly after this time, the small scales saturate and the large-scale magnetic field keeps growing exponentially up to $t=150$.

Although here and in the following discussion we consider quantities averaged over angle in Fourier space to discuss the statistical evolution of kinetic and magnetic energy at different scales, it should be noted that a mean flow appears superimposed upon the turbulent fluctuations, as a result of the nonrandom external forcing. This mean flow should not be expected to be isotropic or homogeneous, as is usually assumed for Kolmogorov's turbulence.

The total kinetic energy spectra (thick lines) and magnetic energy spectra (thin lines) for run $\mathrm{B}^{\prime}$ are shown in Figure 10. Only two kinetic spectra are shown, at times $t=11.4$ and $t=$
181.8. At early times, the magnetic energy spectrum peaks at small scales $(k \approx 9)$, and the spectrum at large scales seems to satisfy a $k^{3 / 2}$ power law, as already observed for the Taylor-Green flow (Ponty et al. 2005), and for other flows as well (Haugen et al. 2004). The magnetic energy increases from $t=11.4$ to $t=$ 181.8 and eventually dominates the kinetic energy at the longest wavelength.

The appearance of these quasi-DC components of the magnetic field seems to have a profound effect on the short-wavelength kinetic spectral components, depressing them by an order of magnitude, as is also visible from the thick dashed line in Figure 10. The most straightforward interpretation is in terms of what is sometimes called the Alfvén effect. The idea is that in incompressible MHD, any nearly spatially uniform, slowly varying 

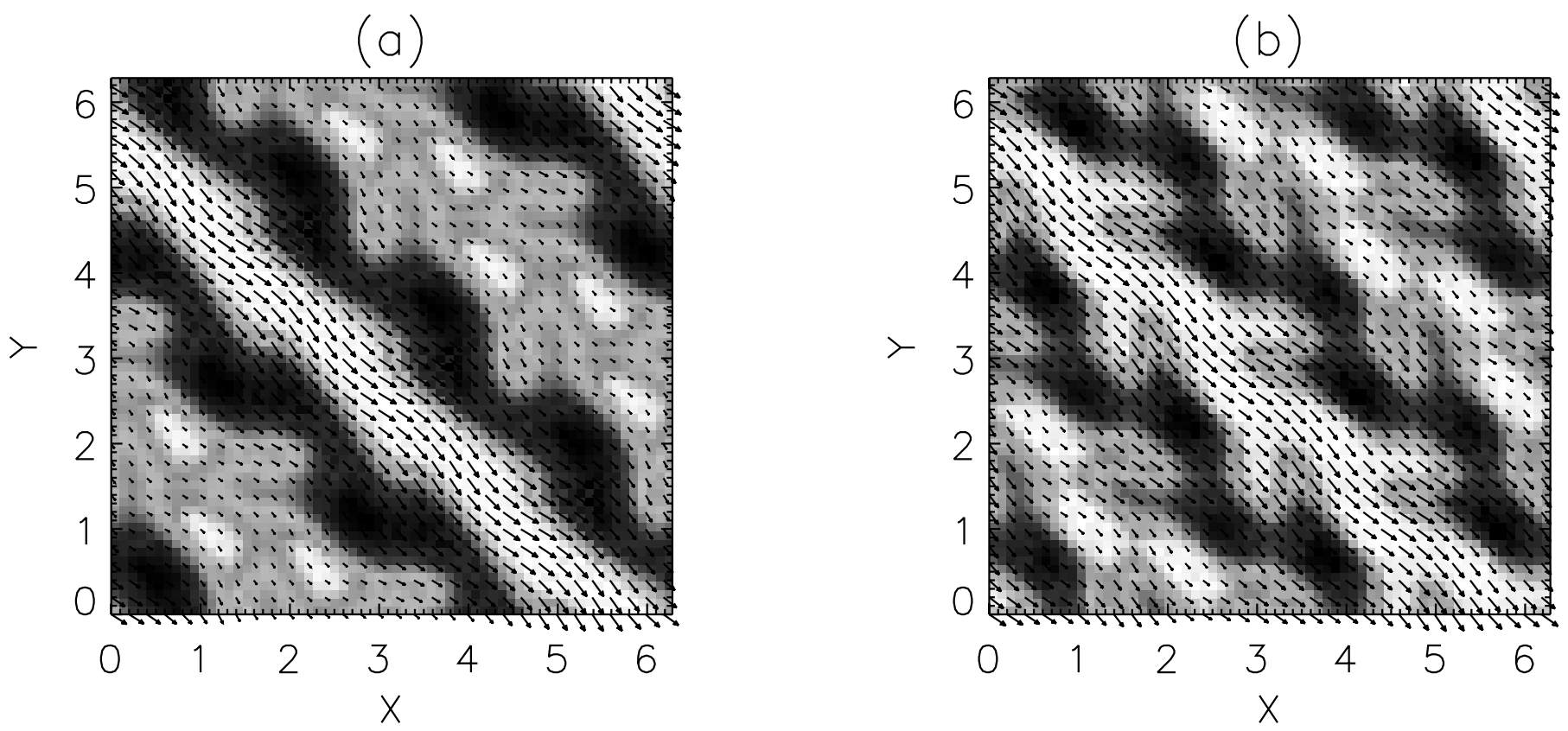

FIG. 7.-Cross-sectional plots of the magnetic field in the plane $z=\pi / 4$ at $(a) t=352$ and $(b) t=368$, for run A. Same conventions as in Fig. 5. [See the electronic edition of the Journal for a color version of this figure.]

magnetic field forces the small-scale excitations to behave like Alfvén waves. In an Alfvén wave, the energy is generally equipartitioned between magnetic field and velocity field, and any mechanism that damps one will damp the other. Since $\eta \gg \nu$ when $P_{M} \ll 1$, the Kolmogorov "inner scale" can be defined entirely in terms of energy dissipation rate and $\eta$, regardless of how much smaller the viscosity is.

This was already observed in closure computations of MHD turbulence at low $P_{M}$ by Léorat et al. (1981). Although in our simulations the magnetic Prandtl number is not small enough to ensure a large-scale separation between the different dissipative scales, to the best of our knowledge this is the first time this effect predicted by closures has been observed in a direct nu-

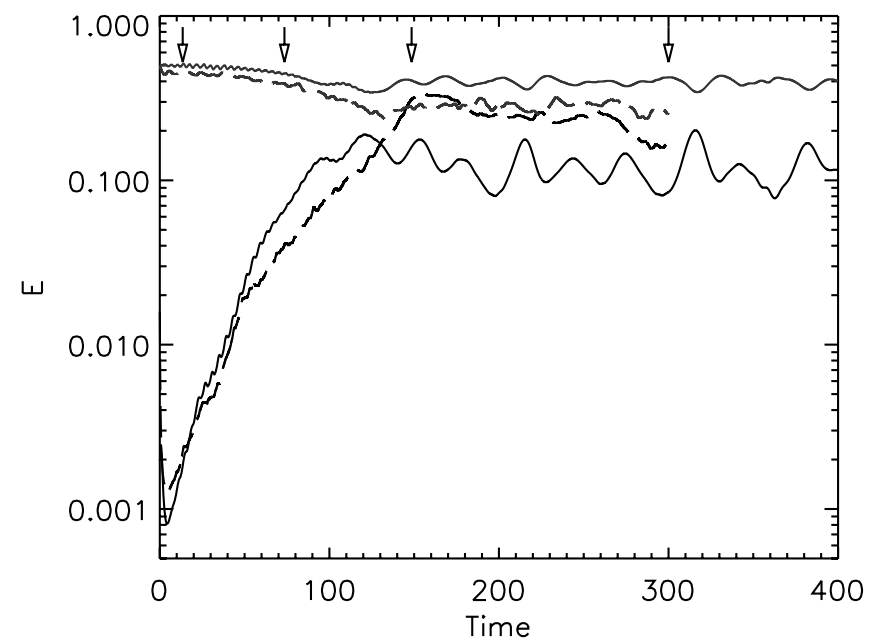

FIG. 8.-Time history of kinetic (upper curves) and magnetic energy (lower curves) for run $\mathrm{A}^{\prime}$ at low Reynolds number (solid lines) and $\mathrm{B}^{\prime}$ at high Reynolds number (dotted lines), both $20 \%$ above threshold. The arrows on the top of the figure represent the times at which the transfer terms displayed in Fig. 11 are evaluated. [See the electronic edition of the Journal for a color version of this figure.] merical simulation. In a previous work (Ponty et al. 2005), the behavior of the dynamo threshold using Taylor-Green forcing from $P_{M}=1$ down to $10^{-2}$ was studied using direct numerical simulations and two independent models of MHD turbulence. At a mechanical Reynolds number of 675 and $P_{M}^{-1}=2.98$, the threshold curve for dynamo action is observed to level off, and an asymptotic regime is reached where the critical magnetic Reynolds number required to have dynamo instability turns out to be independent of $P_{M}$. Simulations B and $\mathrm{B}^{\prime}$ are in the vicinity of this asymptotic regime, corresponding to low values of the

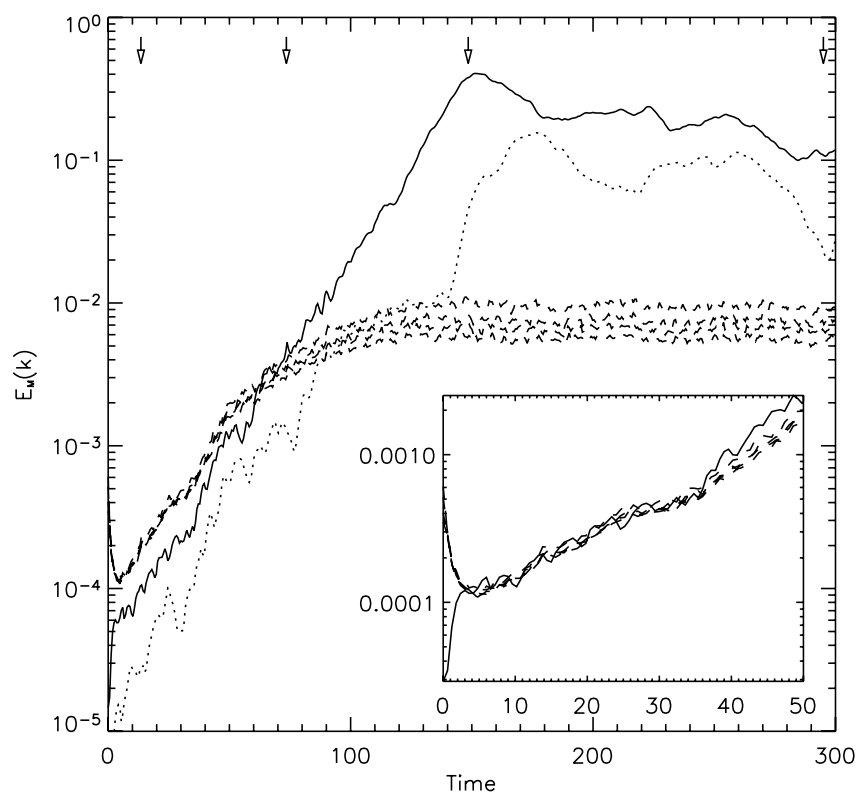

FIG. 9. $-B^{2}$ integrated over different shells in Fourier space as a function of time, for run $\mathrm{B}^{\prime}$ and $k=1$ (dotted line), $k=2$ (solid line), and $k=9,10,11$, and 12 (dashed lines). The inset shows the evolution at early times, with all the shells normalized to have the same amplitude. The arrows are at the same times as in Fig. 8. 


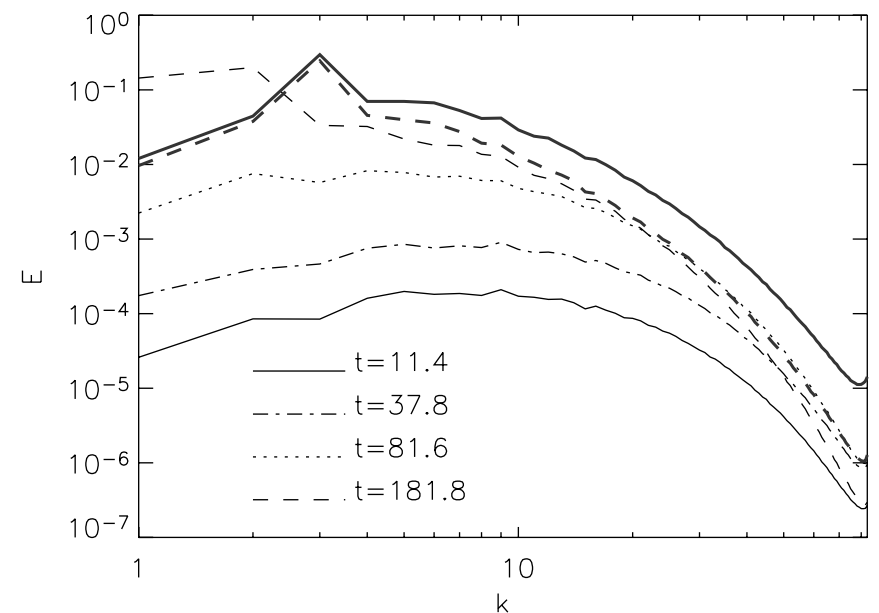

FIG. 10.-Kinetic (thick lines) and magnetic energy spectra (thin lines) as a function of time for run $\mathrm{B}^{\prime}$. Kinetic spectra are only shown at $t=11.4$ and $t=181.8$; note in the latter case, the strong diminution of the kinetic energy spectrum at small scales and its similarity to the magnetic spectrum there. [See the electronic edition of the Journal for a color version of this figure.]

magnetic Prandtl number. For a different external forcing (see, e.g., Schekochihin et al. [2004] and Haugen et al. [2004] for the implications of a purely random and nonhelical force), the asymptotic behavior as a function of $P_{M}$ can differ.

Note that with high Reynolds numbers, in the pure hydrodynamic case, excitations will go farther out in $k$ space. But once a large-scale magnetic field is present, if small scales behave like an approximately equipartitioned Alfvén wave, the larger transport coefficient will drain both the $\boldsymbol{v}$ and $\boldsymbol{B}$ fields (resistivity in this case). One could jump to the conclusion that for $\nu / \eta \ll 1$, the dynamo process will behave as if $P_{M}$ were of $\mathcal{O}(1)$ at all times (see Yousef et al. [2003] for different simulations supporting this conclusion). We warn that this is certainly inappropriate in the formation, or kinematic phase, when the magnetic field is small but amplifying, and there is no quasi-DC magnetic field to enforce the necessary approximate equipartition at small scales. This warning can also apply in more complex systems, such as during the reversals of the Earth's dynamo.

The central role played by the $-\boldsymbol{v} \cdot(\boldsymbol{j} \times \boldsymbol{B})$ term by which energy is extracted from the velocity field can be clarified by plotting the transfer functions $T(k)$ for the magnetic field and velocity field as functions of $k$ at different times.

The energy transfer function

$$
T(k)=T_{V}(k)+T_{M}(k)
$$

represents the transfer of energy in $k$-space and is obtained by dotting the Fourier transform of the nonlinear terms in the momentum equation (2) and in the induction equation (3) by the Fourier transform of $\boldsymbol{v}$ and $\boldsymbol{B}$, respectively. It also satisfies

$$
0=\int_{0}^{\infty} T\left(k^{\prime}\right) d k^{\prime}
$$

because of energy conservation by the nonlinear terms; one can also define

$$
\Pi(k)=\int_{0}^{k} T\left(k^{\prime}\right) d k^{\prime}
$$

where $\Pi(k)$ is the energy flux in Fourier space. In equation (11), $T_{V}(k)$ is the transfer of kinetic energy

$$
T_{V}(k)=\int \hat{\boldsymbol{v}}_{k} \cdot\left[-(\widehat{\boldsymbol{\omega \times \boldsymbol { v }}})_{k}+(\widehat{\boldsymbol{j} \times \boldsymbol{B}})_{k}\right]^{*} d \Omega_{k},
$$

where the hat denotes Fourier transform, the asterisk complex conjugate, and $d \Omega_{k}$ denotes integration over angle in Fourier space. In this equation and the following, it is assumed that the complex conjugate of the integral is added to obtain a real transfer function.

The transfer of magnetic energy is given by

$$
T_{M}(k)=\int \hat{\boldsymbol{B}}_{k} \cdot \nabla \times(\widehat{\boldsymbol{v} \times \boldsymbol{B}})_{k}^{*} d \Omega_{k},
$$

and we can also define the transfer of energy due to the Lorentz force,

$$
T_{L}(k)=\int \hat{\boldsymbol{v}}_{k} \cdot(\widehat{\boldsymbol{j} \times \boldsymbol{B}})_{k}^{*} d \Omega_{k} .
$$

Note that this latter term is part of $T_{V}(k)$; it gives an estimation of the alignment between the velocity field and the Lorentz force at each Fourier shell (as shown previously in Fig. 3). This term also represents energy that is transferred from the kinetic reservoir to the magnetic reservoir [in the steady state, the integral over all $k$ of $T_{L}(k)$ is equal to the magnetic energy dissipation rate, as follows from eq. (3)].

Figure 11 shows the transfer functions $T(k ; t=0)$, which corresponds to the total energy transfer in the hydrodynamic simulation, since the magnetic seed has just been introduced; $T(k)$, which is the total energy transfer; $T_{V}(k) ; T_{M}(k)$; and $-T_{L}(k)$, as functions of $k$ for four different times for run $\mathrm{B}^{\prime}$. A gap in one of the spectra indicates (since the plotting is logarithmic) that it has changed sign. It is apparent that the dominant transfer is always in the vicinity of the forcing band, although it is quite spread over all wavenumbers in the inertial range at all times. It is also apparent that at the later times, most of the transfer is magnetic transfer, in which, of course, the velocity field must participate (see eq. [15]).

During the kinematic regime (Fig. 11a), the kinetic energy transfer $T_{V}(k)$ is almost equal to the total transfer. Note that $-T_{L}(k)$ is approximately constant between $k \approx 3$ and $k \approx 12$; all these modes in the magnetic energy grow with the same growth rate (see Fig. 9). The negative sign of $T_{L}(k)$ shows that energy is being extracted from the velocity field; in physical space the electromagnetic force associated with the currents induced by the motion of the fluid opposes the change in the field in order to ensure the conservation of energy, as follows from Lenz's law. On the other hand, the amplified magnetic field is getting its energy from the velocity field. Note that then $-T_{L}(k)$ can be used as a signature of the scale at which the magnetic field extracts energy from the velocity field [compare this result with the low $R_{V}$ case, where $-T_{L}(k)$ peaks at $k=3$ both in the kinematic regime and in the nonlinear stage]. As its counterpart, the transfer of magnetic energy $T_{M}(k)$ represents both the scales at which magnetic field is being created by stretching and the nonlinear transfer of magnetic energy to smaller scales. The $T_{L}(k)$ is peaked at wavenumbers larger than $T_{M}(k)$; the magnetic field extracts energy from the flow at all scales between $k \approx 3$ and $k \approx 12$, and this energy turns into magnetic energy at smaller scales. Note that this is in agreement with theoretical arguments (Verma 2004) and closures (Pouquet et al. 1976) suggesting that 

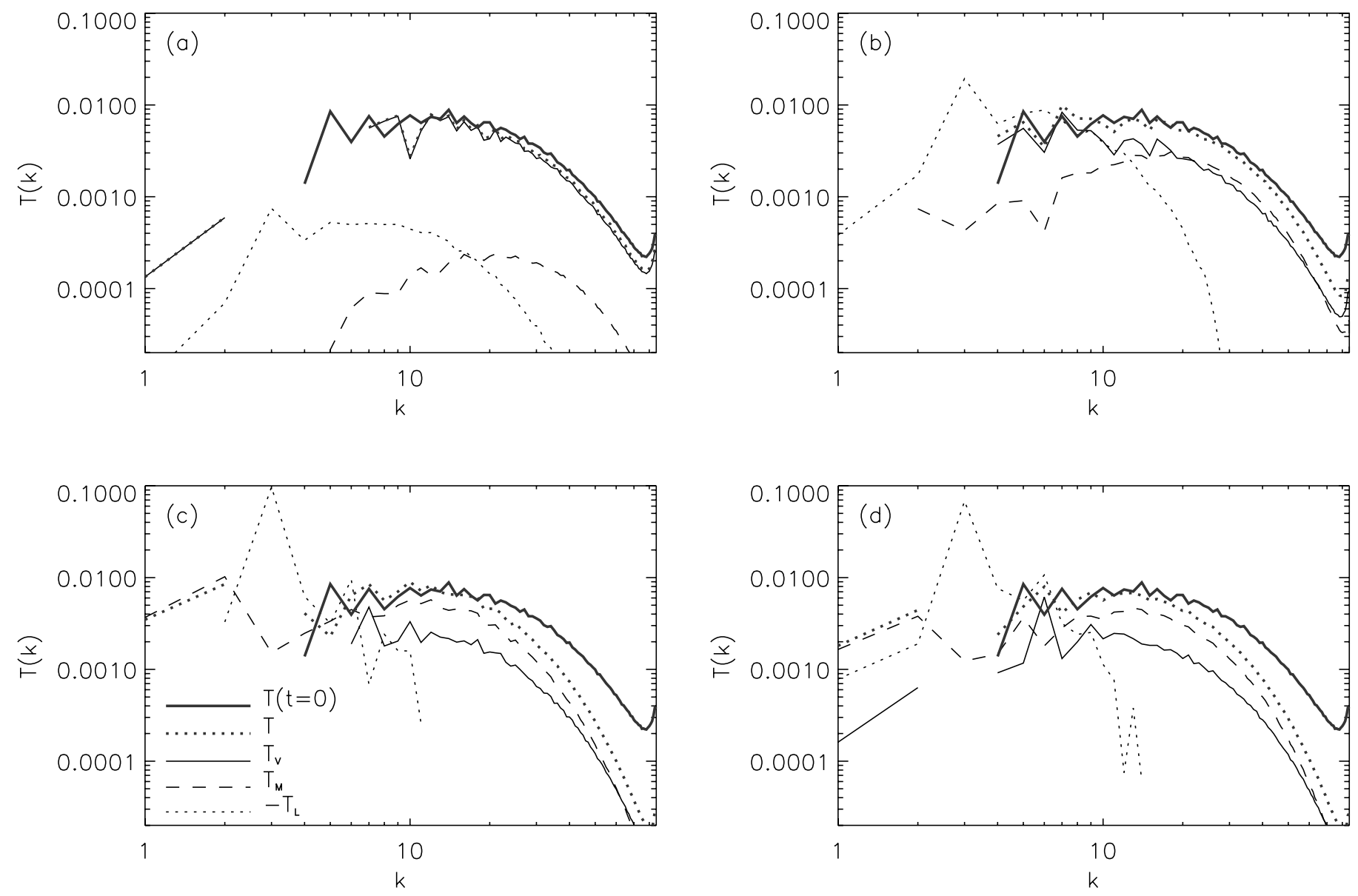

FIg. 11. - Transfer of energy in Fourier space in run $\mathrm{B}^{\prime}$, at $(a) t=13.5$ in the kinematic regime (see Fig. 9), (b) $t=73.5$ at the end of the kinematic regime, (c) $t=148.5$ at the time of rapid growth of the $k=2$ shell, and $(d) t=300$. In all the figures, the total transfer at $t=0$ (thick solid line) is shown as a reference (which corresponds to the $\boldsymbol{B}=0$ case); dotted, dashed, and solid lines represent transfer as indicated in $(c)$. Since $T_{L}$ is negative at most $k,-T_{L}$ is shown. [See the electronic edition of the Journal for a color version of this figure.]

in nonhelical MHD turbulence dominated by kinetic energy, the transfer from $\boldsymbol{v}$ to $\boldsymbol{B}$ can take place nonlocally from large wavenumbers to smaller wavenumbers.

As time evolves and the magnetic small scales saturate, a peak in $-T_{L}(k)$ grows at $k=3$ (Fig. $11 b$ ). At the same time, the transfer of kinetic energy $T_{V}(k)$ at small scales is quenched (compared with Fig. 11a, it has diminished in amplitude by almost 1 order of magnitude). This time corresponds to the time at which a large-scale $(k=2)$ magnetic field starts to grow (see Figs. 9 and 10). In the saturated regime (Figs. $11 c$ and $11 d$ ) $T_{L}(k)$ is negative at large scales and peaks strongly at $k=3$ at late times. A substantial fraction of the injected mechanical energy is seen to be extracted by the magnetic field in the injection band $(k=3)$. As previously mentioned, $T_{M}(k)$ represents energy extracted from the kinetic reservoir, which will be finally injected into the magnetic reservoir (not necessarily at the same scales). Note that $T_{M}(k)$ peaks at $k=2$. This magnetic energy is then carried to small scales by the magnetic field $\left[T_{M}(k)>T_{V}(k)\right.$ up to the diffusion scale in the steady state; Figs. $11 c$ and $11 d]$. A counterpart of this dynamic was observed in Haugen et al. (2004), in which it was noted by examination of global quantities that most of the energy injected in the saturated regime of the dynamo is dissipated by the magnetic field. Similarly, in run B we find that $\left\langle\nu \omega^{2}\right\rangle /\left\langle\eta j^{2}\right\rangle \approx 0.4$ at $t=300$. This is associated with the drop in the kinetic energy spectrum at late times (Fig. 10). Note also that the transfer functions $T_{V}(k)$ and $T_{M}(k)$ drop together at small scales.
In summary, during the kinematic regime the magnetic field is amplified in a broad region of $k$-space, while in the nonlinear phase most of the amplification takes place at large scales. This contrasts with the low $R_{V}$ and $P_{M} \approx 1$ case, where the magnetic energy grows at large scales $(k=2)$ from the beginning of the kinematic dynamo phase, the small scales being undeveloped.

Finally, we may ask if anything remains visible of any pattern enforced by the forcing function $F \boldsymbol{v}_{\mathrm{TG}}$ in the higher Reynolds number runs. Figure 12 suggests that the answer is yes. This figure is a plot for run $\mathrm{B}^{\prime}$ of a cross section $(y=\pi / 4)$ in which the magnetic field strength is exhibited: arrows denote components in the plane and colors denote components normal to the plane. The left panel is at $t=70$, and the right panel is at $t=300$. The two horizontal bands are associated with the stagnation planes of the Taylor-Green forcing.

While at $t=70$ the magnetic field is mostly at small scales, at $t=300$ a pattern reminiscent of the low $R_{V}$ case (Fig. $6 d$ ), albeit more turbulent, can be clearly seen. This is the result of the suppression of small scales by the magnetic field. While in the kinematic regime the magnetic field grows under a broad kinetic energy spectrum, when the large-scale magnetic field starts to grow, the small-scale velocity field fluctuations are quenched (Figs. 10 and 11), and the large-scale pattern reappears. This "cleanup" effect was also observed in Brandenburg et al. (2001), but there both the large-scale pattern in the flow and the turbulent fluctuations at small scale were imposed, while here the turbulent 
(a)

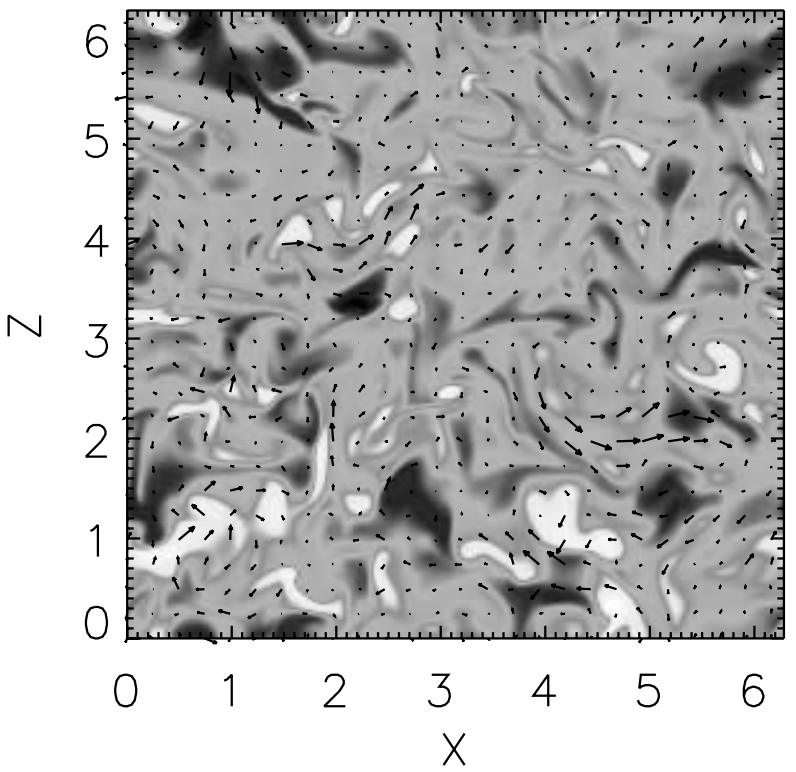

(b)

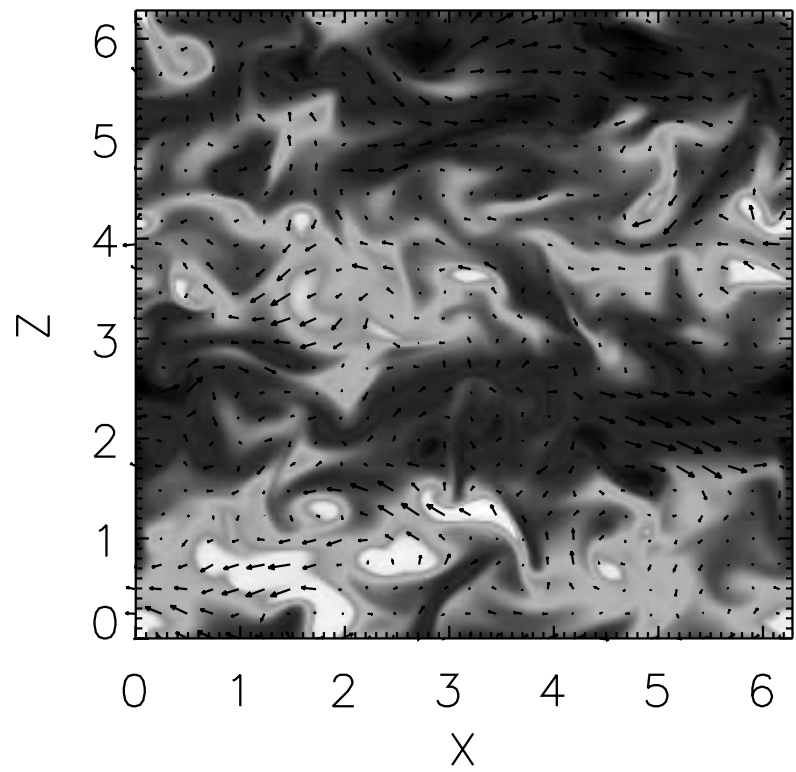

FIG. 12. - Cross-sectional plots of the magnetic field in the plane $y=\pi / 4$, at $(a) t=70$ and $(b) t=300\left(B_{x}, B_{z}\right.$ is indicated by the arrows, and $B_{y}$ by the color) for run $\mathrm{B}^{\prime}$. [See the electronic edition of the Journal for a color version of this figure.]

fluctuations are the result of the large-scale external force and high values of $R_{V}$.

\section{DISCUSSION AND SUMMARY}

The oscillatory behavior exemplified in Figure 2 is not without precedent. It is common for a pulsation to occur in a faucet when the pressure drop is such as to cause the flow of the water to be close to a speed near the threshold of the transition to turbulence. The developing turbulence acts as an eddy viscosity to reduce the Reynolds number back into the laminar regime. As the turbulence then subsides, the flow accelerates until the flow speed is again in the unstable regime, and the cycle repeats. A few years ago (Shan \& Montgomery 1993a, 1993b), a similarly quasi-periodic behavior was observed in an MHD problem, which might be considered an opposite limit of the dynamo problem. A quiescent, periodic circular cylinder of magnetofluid was supported by an external axial magnetic field and carried an axial current driven by an applied axial voltage. By increasing the axial current, it was possible to cross a stability boundary for the onset of mechanical motion. The unstable modes were helical, with regard to the behavior of $\boldsymbol{v}$ and $\boldsymbol{B}$. The resulting $-\boldsymbol{v} \times \boldsymbol{B}$ axial electromotive force opposed the sense of the applied electric field and constituted an effective increase in the resistance of the column. When the disturbances grew large enough, the total axial current was reduced back below the stability threshold, causing the magnetofluid to relaminarize itself. A cyclic oscillation in magnetic and kinetic energy resulted, with the larger energy being magnetic, which in many ways resembles qualitatively the oscillations exhibited in Figure 2, except that the magnetic energy remained larger: a sort of "inverse dynamo" problem.

In the high Reynolds case (runs B and $\mathrm{B}^{\prime}$ ), part of this dynamic persists. The initial kinetic energy spectrum is broader, and velocity field fluctuations are larger. The large-scale flow is still present but is modified by the turbulent fluctuations. In the kinematic regime, the magnetic field grows at all wavenumbers with the same growth rate, and the magnetic energy spectrum is peaked at scales smaller than the integral length scale of the flow. After the nonlinear saturation of the small-scale magnetic field, the field grows at scales larger than the integral scale of the flow. There are no recognizable common features between the magnetic field in the kinematic regime and the magnetic structures observed in the low Reynolds number case. However, in the steady state of the dynamo, the large-scale magnetic field forces small-scale excitations to be equipartitioned between magnetic and velocity fields, and both fields are damped at almost the same scale. As a result, velocity fluctuations are strongly suppressed, and at late times similar structures can be recognized in the magnetic field in both the low and high Reynolds simulations.

By examination of nonlinear transfer functions in Fourier space, interactions at large and small scales between kinetic and magnetic energy were found. During the kinematic regime, the magnetic field extracts energy from the velocity field at intermediate scales, and this energy is turned into magnetic energy at smaller scales. After the saturation and quenching of the small scales, a substantial fraction of the kinetic energy is still extracted from the forcing band, and the magnetic field grows at large scales. In the saturated regime, the magnetic and kinetic energy transfer functions drop at the same scale, although the magnetic transfer is larger than the kinetic one.

It is clear that there are many distinct dynamo behaviors, depending upon the parameters and the nature and scale of the mechanical forcing. It should not be inferred that the oscillatory behavior shown in Figure 2 is more generic than it is. Different regimes can appear as the forcing amplitude is varied, for example, or as the timing of the seed magnetic field's introduction is varied. The dynamics can acquire different qualitative features as these features are changed. It can be expected that, once turbulent computations in geometries other than rectangular periodic ones are undertaken, still further variety may occur.

We thank H. Tufo for providing computer time at University of Colorado, Boulder, NSF ARI grant CDA-9601817. Computer time was also provided by NCAR. The NSF grants ATM-0327533 at Dartmouth College and CMG-0327888 at NCAR supported this work in part and are gratefully acknowledged. J. F. P., H. P., and Y. P. thank the CNRS Dynamo GdR and INSU/PNST Program for support. 


\section{REFERENCES}

Archontis, V., Dorch, S. B. F., \& Nordlund, A. 2003, A\&A, 410, 759

Bourgoin, M., Odier, P., Pinton, J.-F., \& Ricard, Y. 2004, Phys. Fluids, 16, 2529 Brandenburg, A. 2001, ApJ, 550, 824

Brandenburg, A., Bigazzi, A., \& Subramanian, K. 2001, MNRAS, 325, 685

Haugen, N. E., Brandenburg, A., \& Dobler, W. 2004, Phys. Rev. E, 70, 036408

Kazantsev, A. P. 1967, Soviet Phys.-JETP, 26, 1031

Léorat, J., Pouquet, A., \& Frisch, U. 1981, J. Fluid Mech., 104, 419

Marié, L., Burguete, J., Daviaud, F., \& Léorat, J. 2003, European J. Phys. B, 33, 469

Moffatt, H. K. 1978, Magnetic Field Generation in Electrically Conducting Fluids (Cambridge: Cambridge Univ. Press)

Morf, R. H., Orszag, S. A., \& Frisch, U. 1980, Phys. Rev. Lett., 44, 572

Nore, C., Brachet, M. E., Politano, H., \& Pouquet, A. 1997, Phys. Plasmas, 4, 1

Pelz, R. B., Yakhot, V., Orszag, S. A., Shtilman, L., \& Levich, E. 1985, Phys. Rev. Lett., 54, 2505
Ponty, Y., Gilbert, A. D., \& Soward, A. M. 2001, in Dynamo and Dynamics: A Mathematical Challenge, ed. P. Chossat, D. Armbruster, \& I. Oprea (Boston: Kluwer), 261

Ponty, Y., Mininni, P. D., Montgomery, D. C., Pinton, J.-F., Politano, H., \& Pouquet, A. 2005, Phys. Rev. Lett., 94, 164502

Pouquet, A., Frisch, U., \& Léorat, J. 1976, J. Fluid Mech., 77, 321

Taylor, G. I., \& Green, A. E. 1937, Proc. R. Soc. London A, 158, 499

Schaeffer, N. 2004, Ph.D. thesis, Université Joseph Fourier (Grenoble)

Schekochihin, A. A., Cowley, S. C., Maron, J. L., \& McWilliams, J. C. 2004, Phys. Rev. Lett., 92, 054502

Shan, X., \& Montgomery, D. 1993a, Plasma Phys. Controlled Fusion, 35, 619 1993b, Plasma Phys. Controlled Fusion, 35, 1019

Verma, M. K. 2004, Phys. Rep., 401, 229

Yousef, T. A., Brandenburg, A., \& Rüdiger, G. 2003, A\&A, 411, 321 